\title{
Popcorn breeding for water-stress tolerance or for agronomic water-use efficiency?
}

\author{
S.H. Kamphorst, V.J. de Lima, A.T. do Amaral Júnior, \\ K.F.M. Schmitt, J.T. Leite, C.M. Carvalho, R.M.R. Silva, \\ K.B. Xavier, F.R.A. Fereira, P.H.A.D Santos, E. Campostrini \\ Laboratório de Melhoramento Genético Vegetal, Centro de Ciências e \\ Tecnologias Agropecuárias (CCTA), UENF, Campos dos Goytacazes, RJ, \\ Brasil
}

Corresponding author: S. H. Kamphorst

E-mail: samuelkampho@hotmail.com

Genet. Mol. Res. 17 (4): gmr18184

Received October 22, 2018

Accepted December 18, 2018

Published December 26, 2018

DOI http://dx.doi.org/10.4238/gmr18184

\begin{abstract}
Soil water limitations can cause high losses in agricultural yields. In order to investigate how popcorn varieties are affected by reduced water availability, we evaluated grain yield (GY) and popping expansion (PE) of 20 popcorn lines under water stress (WS) and well-watered conditions (WW), to propose discrimination with regard to the level of water-stress tolerance (T) and agronomic water-use efficiency (WUE), as well as to identify crosses for the breeding of superior hybrid combinations and for inheritance studies. A randomized complete block design with three replications was used. Irrigation was applied at pre-anthesis. The germplasm was discriminated based on the Stress tolerance index (STI), Stress susceptibility index (SSI), Stability index (SIN), Drought resistance index (DRI), and Agronomic water-use efficiency (WUE). Genetic diversity was measured by 15 EST-SSR markers. The reduction in GY under water stress was $55.29 \%$ and PE was reduced $29.19 \%$. For the identification of genotypes with higher phenotypic means, STI and WUE were similarly efficient, whereas SSI and SIN identified genotypes with a lower proportional performance loss in the WS compared to the WW environment. For both GY and PE, there was a lack of relationship between WUE (more productive) and $\mathrm{T}$ (more
\end{abstract}


stable). To explore the allelic complementarity for WUE, for T and for both, respectively, the combinations L59 x P7, L55 x P1 and L71 $x$ P6 had the best performances. The lines L61, L63 and L65 phenotypically contrasted to those with high WUE and T and could be used for inheritance studies. Genotypes with higher WUE are considered the most appropriate option for breeding programs under WS.

Key words: Water stress tolerance; Agronomic water-use efficiency; Popping expansion

\section{INTRODUCTION}

When water is limiting in the soil (drought), corn grain yield is extremely low (Cairns et al., 2012; Zia et al., 2013; Adebayo et al., 2014; Mageto et al., 2017; Dias et al., 2018). This meteorological condition occurs worldwide, particularly in tropical areas (Mageto et al., 2017; Sayadi Maazou et al., 2016), including in Brazil (Mendes et al., 2017; Dias et al., 2018). In the future, the imbalance of temporal and spatial distribution of water resources is expected to be aggravated and, along with the temperature rise, to cause increasing losses in agricultural yields of many crops (Suzuki et al., 2014; Zandalinas et al., 2018). In the near future, crop cultivation in environments with constant restrictions and at marginal locations not normally suited for agricultural zoning will become unavoidable (Giaveno et al., 2007; Peters et al., 2011).

Plant breeding focused on water deficiency can exploit different strategies, including developing cultivars with increased water-stress tolerance or agronomic efficiency of water use (WUE). The concept of tolerance is defined as the plant's ability to support, survive and reproduce under stress conditions (Maia et al., 2011). From an agronomic point of view, tolerance $(\mathrm{T})$ can be defined as the ratio of crop yield produced under stress compared to yield under ideal cultivation conditions (Mitra, 2001; Miti et al., 2010). In turn, WUE is related to the greater ability of a genotype to produce under low water availability and is computed as the ratio between grain yield $(\mathrm{kg})$ per unit of available water resource ( $\mathrm{L}$ of applied, transpired or evapotranspired water (Tambussi et al., 2007; Jákli et al., 2018).

Since crops are subject to water stress events (WS), farmers need cultivars that produce satisfactory yields even under this condition, but which, under optimum cultivation conditions, will also produce high yields. Thus, for maize, genotypes with higher productivity tend to have a better grain yield performance, both under adequate cultivation conditions and under WS (Kamara et al., 2003; Zia et al., 2013). However, the most productive genotypes under both environmental conditions (higher use efficiency of water resources) are not the most tolerant, since the proportional losses among the environments are usually higher(Maia et al., 2011; Naghavi et al., 2013).

Several indices have been used to select genotypes with water-stress tolerance and/or agronomic water-use efficiency (those with highest yields), based on the means of the trait under ideal and stress cultivation conditions. The indices can measure the amount of water required to produce a given agronomic characteristic (WUE) in relation to the percentage of losses of the genotype per se in comparison with the yield under ideal conditions; or in relation to the mean of the set of evaluated genotypes; or these factors taken together.

For popcorn, a highly appreciated crop with high economic profitability that moves around US \$ 2 billion annually in the US, no specific research addressing the selection of 
genotypes adapted to WS, greater WS tolerance or higher WUE is available. In this context, in particular with regard to the active popcorn germplasm bank of the State University of Northern Rio de Janeiro (UENF), containing lines of temperate and tropical climates, two questions can be formulated: i) is there genetic variability for water-stress tolerance (T) and/or agronomic water-use efficiency (WUE) to establish lines of research for the breeding program with a view to develop cultivars? ii) how are T and WUE in popcorn genotypes related to the two main traits, i.e., grain yield (GY) and popping expansion (PE)?

In this context, we evaluated various UENF popcorn lines, regarding the grain yield potential (GY) and popping expansion (PE) under WS and WW conditions, to propose discrimination of the genetic merit of WS tolerance and WUE, based on the traits GY and PE, in an attempt to make genotypes of interest available for breeding. Secondly, this study proposed crosses between lines with high genetic merit with a view to establish superior hybrid combinations, as well as to select genotypes for inheritance studies.

\section{MATERIAL AND METHODS}

\section{Plant material, experimental design and cultural treatments}

Twenty popcorn $\left(S_{7}\right)$ lines were evaluated, including germplasm with tropical climate adaptation (L61, L63, L65, L69, L70, and L71 - derived from population 'BRS-Angela') and temperate/tropical germplasm (P1, P5, P6 and P7 - derived from the commercial hybrid 'Zélia'; P2 and P3 - from compound CMS- 42; P4 - from South American races; P8 and P9 - L55, L55 and L59 - from population 'Beija-Flor'; and L75 and L76 - derived from population 'Viçosa').

The lines were separated into two maturation groups, based on the date of male flowering, and each group contained 10 genotypes, namely: i) early (L54, L59, P2, P3, P4, P5, P6, P7, P8, and P9) and late (L55, L61, L63, L65, L69, L70, L71, L75, L76, and P1). The genotypes were classified in phenological groups, based on tests of previous years. To synchronize the phenological stage of male flowering, sowing was staggered, with the first sowing date on April 19 (late genotypes) and the second on April 25 (early genotypes), in 2016.

The experiments were carried out at the Experimental Station of the State Agricultural College Antônio Sarlo, in Campos dos Goytacazes, Rio de Janeiro, Brazil (lat. $21^{\circ} 42$ '48 "S, long. $41^{\circ} 20^{\prime} 38^{\prime \prime} \mathrm{W}, 14 \mathrm{~m}$ asl) in the dry season (from April to August). The experiment was arranged in a randomized complete block design with three replications per water regime: irrigation to field capacity (WW- water regime 1) and under water stress (WS - water regime 2).

Each plot consisted of four 4.4 -m rows, at a plant spacing of $0.20 \mathrm{~m}$ and row spacing of $0.80 \mathrm{~m}$ (23 plants per row). The plot area was defined as $8.00 \mathrm{~m}^{2}$ of the central rows. Fertilization at sowing consisted of $30 \mathrm{~kg} \mathrm{ha}^{-1} \mathrm{~N}$ (as urea), $60 \mathrm{~kg} \mathrm{ha}^{-1} \mathrm{P}_{2} \mathrm{O}_{5}$ (triple superphosphate) and $60 \mathrm{~kg} \mathrm{ha}^{-1} \mathrm{~K}_{2} \mathrm{O}$ (potassium chloride). Side dressing was applied 30 days after sowing, according to the planting scheduling mentioned above, consisting of $100 \mathrm{~kg} \mathrm{ha}^{-1} \mathrm{~N}$ (as urea).

For the different water regimes, irrigation was applied with a drip system (flow rate of $2.3 \mathrm{~mm} \mathrm{~h}^{-1}$ ). One Katif dripper per plant was installed, at a plant spacing of $0.20 \mathrm{~m}$ and row spacing of $0.80 \mathrm{~m}$. Specific irrigation $(\mathrm{mm})$ was applied during the entire cultivation period, in both the irrigated (WW) and water-stressed (WS) areas, in days after sowing (DAS) (Table 1). In the WS area, irrigation was suspended at 49 DAS until harvest. However, at 56, 70, 77, 105, 112, and 119 DAS there was rainfall, totaling $133 \mathrm{~mm}$ (Table 1). Rain events were recorded at a weather station near the experiment. The total water amount applied to the early and latematuration genotypes was $60 \mathrm{~mm}$ in the WS treatment, while WW plants were watered with a total amount of $138 \mathrm{~mm}$. 
To ensure effective water stress, irrigation was interrupted at the pre-flowering phenological stage, precisely, 15 days before male flowering (June 10, 2016). Mean male

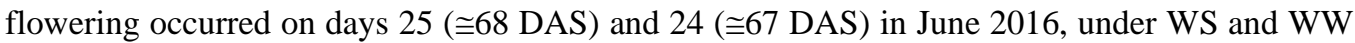
conditions, respectively (data not shown). Throughout the entire crop growth and development cycle, temperature and relative humidity varied from $12-37^{\circ} \mathrm{C}$ and $23-97 \%$, respectively, with a mean solar radiation of $20.35 \mathrm{MJ} \mathrm{m}^{-2} \mathrm{day}^{-1}$ (maximum radiation at the experimental site was $\cong$ $1300 \mu \mathrm{mol} \mathrm{m}^{-2} \mathrm{~s}^{-1}$ (Fig. 1).

Table 1. Precipitation and irrigation ( $\mathrm{mm}$ ) applied to early and late genotypes underwell-watered (WW) and water stress (WS)conditions, in days after sowing (DAS).

\begin{tabular}{|c|c|c|c|c|c|}
\hline DAS & Rainfall & WS - late & WS - early & WW - late & WW - \\
\hline 7 & - & 13 & 13 & 11 & 12 \\
\hline 14 & 26 & 9 & 8 & 9 & 8 \\
\hline 21 & 7 & 8 & 9 & 10 & 11 \\
\hline 28 & 7 & 12 & 10 & 11 & 6 \\
\hline 35 & - & 4 & 6 & 4 & 6 \\
\hline 42 & 1 & 13 & 13 & 14 & 13 \\
\hline 49 & 18 & 2 & 1 & 3 & 3 \\
\hline 56 & 20 & - & - & 4 & 4 \\
\hline 63 & - & - & - & 10 & 10 \\
\hline 70 & 15 & - & - & 9 & 8 \\
\hline 77 & 4 & - & - & 7 & 7 \\
\hline 84 & - & - & - & 14 & 14 \\
\hline 91 & - & - & - & 13 & 12 \\
\hline 98 & - & - & - & 13 & 13 \\
\hline 105 & 24 & - & - & 8 & 10 \\
\hline 112 & 4 & - & - & - & - \\
\hline 119 & 7 & - & - & - & - \\
\hline Total & 133 & 60 & 60 & 138 & 138 \\
\hline
\end{tabular}

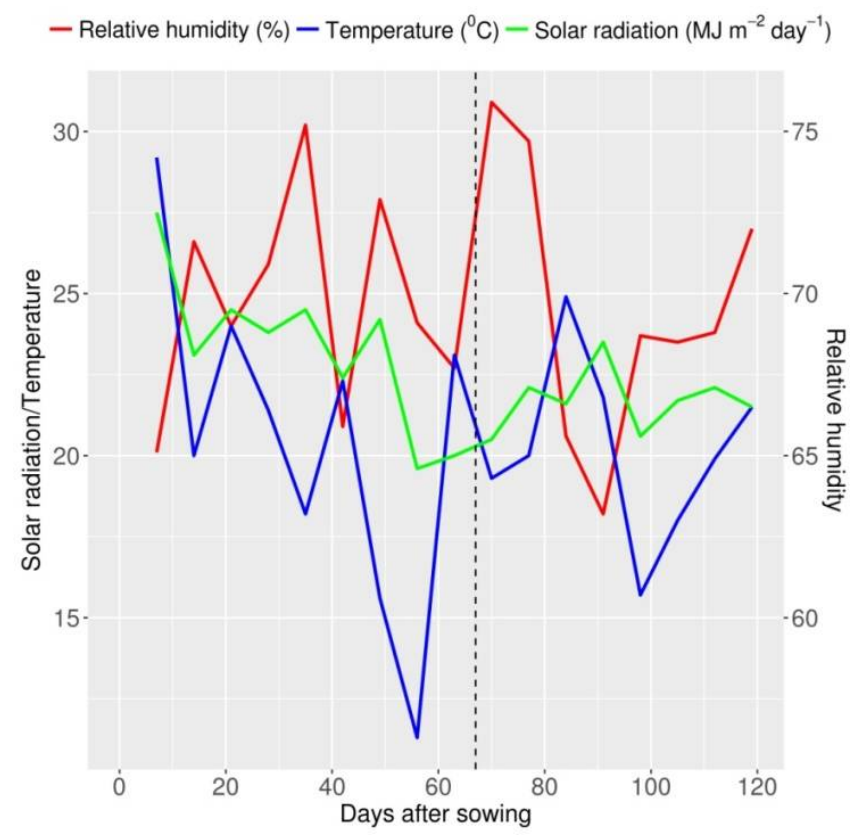

Figure 1. Mean weekly values, in days after sowing, of the temperature $\left({ }^{\circ} \mathrm{C}\right)$, relative humidity $(\%)$ and solar radiation $\left(\mathrm{MJ} \mathrm{m}^{-2}\right.$.day ${ }^{-1}$ ) throughout the growth period of the popcorn lines (April to August 2016). Male flowering began 67 days after sowing (June 24). 
The soil at the experimental station is classified as Yellow Dystrophic Latosolic Fragipan Argisol and, in terms of physical properties (texture), contains high clay and silt levels. The field capacity and permanent wilting points are 38.21 and $28.41 \%$, respectively. During the experiments, the soil water potential was monitored with MPS-6 dielectric water potential sensors, Decagon Devices. In the WS environment, data were sampled at three points, while in the WW environment, at one sampling point. Tensiometers were installed between plants, at a depth of $0.20 \mathrm{~m}$. Soil from the WW environment was maintained at field capacity (-0.01 MPa) throughout the crop cycle, while in the WS environment, the soil reached the permanent wilting point (-1.5 MPa) 12 days after male flowering (Fig. 2).

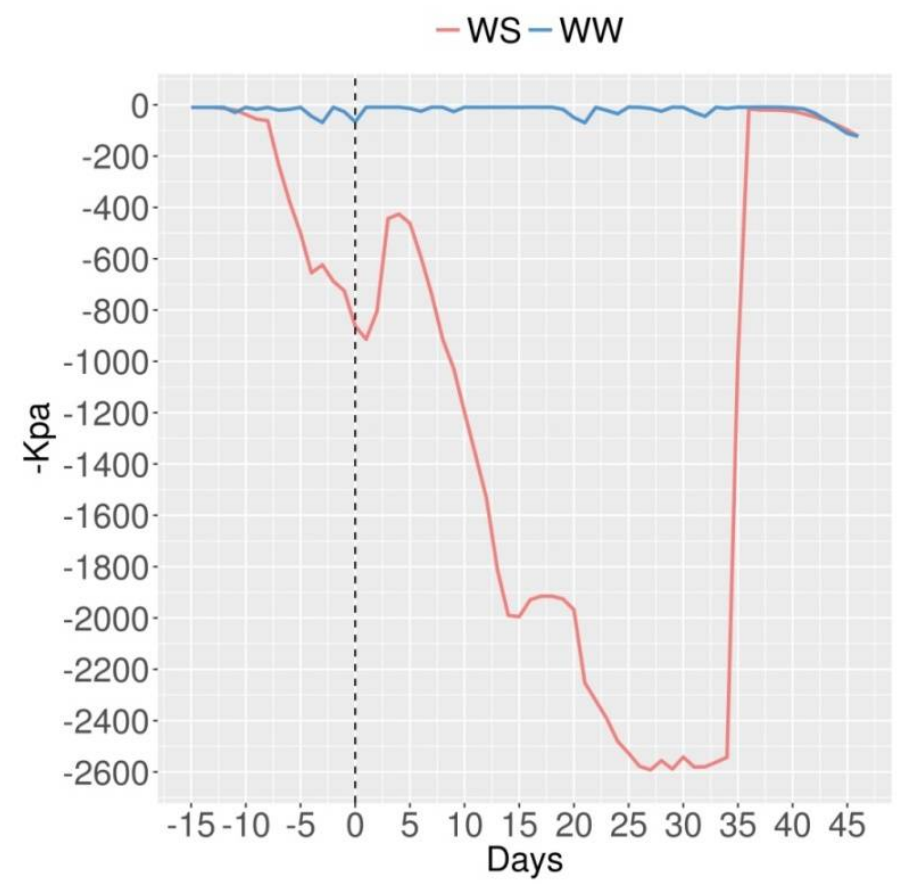

Figure 2. Soil water potential (-KPa) of WW and WS during irrigation suspension. Male flowering began 67 days after sowing (June 24) and is represented on day zero.

\section{Evaluated traits}

Grain yield (GY) was corrected to a moisture content of $13 \%$ and expressed in $\mathrm{Kg}_{\mathrm{K}} \mathrm{ha}^{-1}$. The popping expansion (PE) of $30 \mathrm{~g}$ kernels in a popping paper bag was determined after microwave irradiation at $1000 \mathrm{~W}$, for a fixed time of $2 \min 15 \mathrm{sec}$. Popcorn volume was measured in a graduated beaker $(2,000 \mathrm{~mL})$. Popping expansion (PE) was computed as the quotient of the obtained popcorn volume by grain weight, expressed in $\mathrm{mL} . \mathrm{g}^{-1}$. The trait measurements included all plants of the plot area. 


\section{Statistical analysis}

Individual analyses of variance were carried out for each WW and WS environment, and combined analysis of variance of the experiments, to study the genotype interaction. The analysis was based on the following statistical model: $Y_{i j}=\mu+G_{i}+B_{j}+$ $\varepsilon_{i j}$, where $\mathrm{Y}_{\mathrm{ij}}$ is the observation of the $\mathrm{i}^{\text {th }}$ genotype of the $\mathrm{j}^{\text {th }}$ block; $\mu$ is the general constant; $\mathrm{G}_{\mathrm{i}}$ the treatment effect; $\mathrm{B}_{\mathrm{j}}$ the block effect; and $\varepsilon_{i j}$ the experimental error. Combined variance analysis was performed according to the statistical model: $Y_{i j k}=\mu+G_{i}+$ $B / A_{j k}+A_{j}+G A_{i j}+\varepsilon_{i j k}$, where: $Y_{i j k}$ : observation of the $\mathrm{i}^{\text {th }}$ genotype in the $\mathrm{j}^{\text {th }}$ environment in the $\mathrm{k}^{\text {th }}$ block; $\mu$ : general constant $\mathrm{G}_{\mathrm{i}}$ : random effect of the $\mathrm{i}^{\text {-th }}$ genotype; $B / A_{j k}$ : effect of the $\mathrm{k}^{\text {th }}$ block within environment $\mathrm{j} ; A_{j}$ : fixed effect of the $\mathrm{j}^{\text {th }}$ environment with NID; $G A_{i j}$ : random effect of the interaction between the $\mathrm{i}^{\text {th }}$ genotype with the $\mathrm{j}^{\text {th }}$ environment; and $\varepsilon_{i j k}$ : mean experimental random error associated with observation $Y_{i j k}$, with NID $\left(0, \sigma^{2}\right)$.

When a significant effect of the genotype - environment interaction (GE) was detected, the interaction type was partitioned into simple or complex parts, based on the environmental dissimilarity method proposed by Cruz and Castoldi (1991).

The treatment means were grouped by the Scott and Knott test at 5\% probability.

\section{Mathematical equations for calculating the indices}

The trait mean for the $\mathrm{i}^{\text {th }}$ line $\left(Y_{i}\right)$, at the different water levels, denominated WS-water stress and WW-well-watered was considered for the calculation of the indices. The overall trait mean of each environment was designated $\bar{Y}$. The calculated indices were:

Stress tolerance index (Fernandez, 1992) : $S T I_{i}=Y_{W W_{i}} \times Y_{W S_{i}} / \bar{Y}_{W W}{ }^{2}$

$\left.\bar{Y}_{W S_{i}}\right)$

Stress susceptibility index (Fischer, 1978) : $S S I_{i}=\left(1-Y_{W W_{i}} \times Y_{W S_{i}}\right) /\left(1-\bar{Y}_{W W_{i}} \times\right.$

Stability index (Bouslama and Schapaugh, 1984) : $\operatorname{SIN}_{i}=Y_{W S_{i}} / Y_{W W_{i}}$

Drought resistance index (Blum, 1988):DRI $I_{i}=Y_{W S_{i}} \times\left(Y_{W S_{i}} / Y_{W W_{i}}\right) /\left(\bar{Y}_{W S_{i}}\right)$

Agronomic water-use efficiency: $W U E=\left(Y_{W s_{i}} / L_{W S}+Y_{W W_{i}} / L_{W S}\right) / 2$; where $\mathrm{L}=$ liters of water (precipitation + irrigation).

\section{Euclidean distance, WARD grouping method and principal component analysis}

For the calculation of the Euclidean distance matrix, the estimated mean for each index was standardized by the expression: $z_{i k}=X_{i k} / \hat{\sigma}_{k}$; where $X_{i k}$ corresponds to the mean of the $\mathrm{i}^{\text {th }}$ treatment for the $\mathrm{k}^{\text {th }}$ index and $\hat{\sigma}_{k}$ corresponds to the standard deviation of the $\mathrm{k}^{\text {th }}$ index.

To calculate the distance matrix, the Euclidean distance was used based on the inference: $d_{i i^{\prime}}=\sqrt{\frac{1}{v} \sum_{k=1}^{v}\left(z_{i k}-z_{i^{\prime} k}\right)^{2}}$, where $v$ is the number of studied traits.

The relative importance of the indices used for clustering by the Euclidean distance was determined by the principal component analysis (PCA), in which a higher and lower 
relevance of the indices and/or associations between them was identified by the correlation matrix between the original data (Cruz et al., 2012). The standardized mean was also used for principal component analysis.

\section{Genotyping}

\section{DNA extraction}

The plant material was derived from two seeds of each line, germinated in a tray, containing commercial substrate. After seven days, young leaves were collected for DNA extraction, according to the standard CTAB method (Doyle and Doyle, 1990), with modifications suggested by Daher et al. (2002). The DNA samples were quantified with a Qubit ${ }^{\circ} 2.0$ fluorimeter (Invitrogen). The study samples were diluted to a concentration of $10 \mathrm{ng} / \mu \mathrm{L}$.

\section{Microsatellite markers and polymerase chain reaction (PCR)}

The step of selection of genetic microsatellite markers (EST-SSR) was initiated with 193 primers. Sequences had been developed and mapped earlier by Sharopova et al. (2002) for this species.

Each PCR had a final volume of $13 \mu \mathrm{L}$, consisting of: $2 \mu \mathrm{L}$ DNA $(5 \mathrm{ng} / \mu \mathrm{L}), 1.5$ $\mu \mathrm{L} 10 \mathrm{X}$ buffer $\left(\mathrm{NH}_{4} \mathrm{SO}_{4}\right), 1 \mu \mathrm{LM}_{\mathrm{g}} \mathrm{Cl}_{2}(25 \mathrm{nM}), 1.5 \mu \mathrm{L}$ dNTPs $(5 \mu \mathrm{M})$, and $0.12 \mu \mathrm{lTaq}-$ DNA polymerase $(5 \mathrm{U} / \mu \mathrm{L})$ (Invitrogen, Carlsbad, California, USA).

Polymerase chain reactions were programmed for 35 cycles in an Applied Biosystems/Veriti ${ }^{\circledR}$ 96-Well Thermal Cycler. Each cycle consisted of the following steps: initial denaturation at $94^{\circ} \mathrm{C}$ for $4 \mathrm{~min}$; cyclic denaturation at $94^{\circ} \mathrm{C}$ for $1 \mathrm{~min}$; annealing - specific temperature of each primer in ${ }^{\circ} \mathrm{C}$ for $1 \mathrm{~min}$ (Pena et al., 2016); cyclic extension at $72^{\circ} \mathrm{C}$ for $2 \mathrm{~min}$ and; final extension at $72^{\circ} \mathrm{C}$ for $7 \mathrm{~min}$.

The PCR amplification products were separated by electrophoresis in $4 \%$ Metaphor agarose gels immersed in buffer tanks with TAE $(90 \mathrm{mM}$ Tris-Acetate $(\mathrm{pH} 8.0)+10 \mathrm{mM}$ EDTA). The PCR products were stained with Gel Red ${ }^{\mathrm{TM}}$ and Blue Juice (1:1) for separation. The gels were visualized and documented in a MiniBis Pro system (Bio-Imaging Systems). The amplification product was compared to a $100 \mathrm{bp}$ High DNA Mass Ladder $(0.5 \mathrm{ng} / \mu \mathrm{L})$ marker (Invitrogen, USA).

From the tested primer set, a total of 15 pairs were polymorphic (Table 2) and thus selected for resolution in a capillary electrophoresis fragment analyzer (Advanced Analytical). To this end, $4 \mu \mathrm{l}$ material from the previous amplification was used, together with $20 \mu \mathrm{l}$ buffer, distributed in equipment-specific plates. The amplified fragment size was determined with a known (35 - $500 \mathrm{bp)} \mathrm{molecular}$ weight marker (DNA Ladder). 
Table 2.Sequence of 15 pairs of SSR-EST primers, chromosome location $(\mathrm{Cr})$, position in the genetic map in centimorgans $(\mathrm{cM})$, annealing temperature $\left(\mathrm{T}^{\circ} \mathrm{C}\right)$, and number of alleles $(\mathrm{Na})$.

\begin{tabular}{|c|c|c|c|c|c|}
\hline Locus & Sequence $\left(5^{\prime} \square 3^{\prime}\right)$ & $\mathrm{Cr}$ & $\mathbf{c M}$ & $\mathbf{T}^{\circ} \mathbf{C}$ & $\mathbf{N a}$ \\
\hline \multirow{2}{*}{ umc1073 } & CACCAACGCCAATTAGCATCC & \multirow{2}{*}{1} & \multirow{2}{*}{207} & \multirow{2}{*}{64} & \multirow{2}{*}{2} \\
\hline & GTGGGCGTGTTCTCCTACTACTCA & & & & \\
\hline \multirow{2}{*}{ umc2112 } & AGCTCTACCAAACACGAGCTTCAT & \multirow{2}{*}{1} & \multirow[t]{2}{*}{360} & \multirow[t]{2}{*}{63} & \multirow[t]{2}{*}{3} \\
\hline & CAAATGCAGAAAGATAACGCGAAT & & & & \\
\hline \multirow{2}{*}{ umc1515 } & AGAGAGGCTGCTTCAATAAGTTGC & \multirow{2}{*}{1} & \multirow{2}{*}{393} & \multirow{2}{*}{62} & \multirow{2}{*}{3} \\
\hline & TTAGTAGTTTCGGTGTCCGTTTCC & & & & \\
\hline \multirow{2}{*}{$g l b l$} & GCACACACACAGGACGACAGT & \multirow{2}{*}{1} & \multirow{2}{*}{749} & \multirow{2}{*}{62} & \multirow{2}{*}{3} \\
\hline & TGTTGCTCGGTCACCATACC & & & & \\
\hline \multirow{2}{*}{ umc1118 } & ATCAGATTCCGAAGGGTCCATAAT & \multirow{2}{*}{1} & \multirow{2}{*}{902} & \multirow{2}{*}{62} & \multirow{2}{*}{2} \\
\hline & GTAGTGAAATGAATCGTGAGAGCG & & & & \\
\hline \multirow{2}{*}{ phi96100 } & AGGAGGACCCCAACTCCTG & \multirow{2}{*}{2} & \multirow{2}{*}{24} & \multirow{2}{*}{59} & \multirow{2}{*}{5} \\
\hline & TTGCACGAGCCATCGTAT & & & & \\
\hline \multirow{2}{*}{ olel } & AGTAAAAGAGGCAAGGACTACGGC & 2 & 203 & 62 & 3 \\
\hline & GCGGCGATATATACGAGGTTGT & & & & \\
\hline umc1252 & CTTCTGCATCATCATCATCGTCTT & 2 & 517 & 63 & 3 \\
\hline & GCGTCGGAGAAGTACATCAAGTTT & & & & \\
\hline bip 2 & AGCAAGCAGTTCGAAACAAGGAT & 4 & 669 & 64 & 3 \\
\hline & GACACCAGCACCACTTGAACG & & & & \\
\hline umc 2319 & GCTCTCACTAGCCTCGCATTCC & 6 & 245 & 64 & 3 \\
\hline & GATCCACGCGAGGTTCACTG & & & & \\
\hline$y 1$ & CAAGAAGAGGAGAGGCCGGA & 6 & 99.4 & 65 & 3 \\
\hline & TTGAGCAGGGTGGAGCACTG & & & & \\
\hline umc1241 & TGAAGCAAGTCACTGGTAAGAGCA & 7 & 13.8 & 63 & 2 \\
\hline & TGACACACCCATACTTCCAACAAG & & & & \\
\hline umcllas & TTTGTAATATGGCGCTCGAAAACT & 8 & 26.8 & 63 & 2 \\
\hline & GAAGACGCCTCCAAGATGGATAC & & & & \\
\hline umcl130 & TTGGGACTCATTACTTCCGGACT & 8 & 293 & 63 & 2 \\
\hline & GCTAGGGGAAAGCTCGTACTATGG & & & & \\
\hline umc1982 & AATCGTACTTGGAGGAGGCGTT & 9 & 582 & 63 & 3 \\
\hline & TTCATCTTCCTAGTCTCGTCTCCG & & & & \\
\hline
\end{tabular}

\section{Molecular data analysis}

The genotypic data of each SSR locus were considered as multi-random variables, for the calculation of the genetic distance, using the Gower algorithm (Gower, 1971). This distance matrix was represented as a heatmap using the gplots $\mathrm{R}$ package. Molecular data were analyzed by R language (R Core Team, 2017), with the "cluster" package (Maechler et al., 2017), to calculate the genetic distance and gplots for the heat map (Warnes et al., 2016).

\section{RESULTS}

\section{Genotypic expression of popcornlines in WS and WW environments}

In the WS and WW environments, genetic variability was found among the evaluated genotypes for the agronomic traits grain yield (GY) and popping expansion (PE) 
(Table 3), statistically significant by the F test ( $\mathrm{p}<0.01)$. The reduction in GY was $55.29 \%$, given the overall means of the WS and WW environments of $1139.11 \mathrm{Kg}^{-h^{-1}}{ }^{-1}$ and 2548.08 $\mathrm{Kg} \mathrm{ha}^{-1}$, respectively. Popping expansion under WS was $20.91 \mathrm{~mL} . \mathrm{g}^{-1}$ and $29.35 \mathrm{~mL} . \mathrm{g}^{-1}$ in WW, corresponding to a reduction of $29.19 \%$. In the combined analysis, for the sources of variation genotypes and environments, the traits GY and PE indicated differences among genotype under the different water regimes ( $p$ <.01) (Table 3). An effect of the GE interaction was observed on the evaluated traits.

The environmental dissimilarity analysis detected simple type interaction for GY, explaining $78.69 \%$ of the variation in the genotype mean square (GMS). For trait PE, only $22.41 \%$ of the interaction was attributed to the simple type, while the complex interaction type had the greatest effect.

Table 3. Summary of variance analysis, environmental dissimilarity and over all means and trait means of popcorn lines under water stress (WS) and well-watered (WW) conditions.

\begin{tabular}{|c|c|c|c|c|}
\hline \multirow{2}{*}{\multicolumn{2}{|c|}{ Source of variation }} & \multirow{2}{*}{ DF } & GY & $\mathrm{PE}$ \\
\hline & & & \multicolumn{2}{|c|}{ MS } \\
\hline \multirow{5}{*}{ 戠 } & Gen & 19 & $559525.58^{28}$ & $36.40^{* m}$ \\
\hline & Rep & 2 & 137418.51 & 2.76 \\
\hline & Residuals & 38 & 66624.41 & 3.35 \\
\hline & $\mathrm{CV}(\%)$ & & 22.66 & 8.75 \\
\hline & Mean & & 1139.12 & 20.91 \\
\hline \multirow{5}{*}{ 莺 } & Gen & 19 & $1839610.00^{* *}$ & $52.27^{* m}$ \\
\hline & Rep & 2 & 601287.53 & 2.53 \\
\hline & Residuals & 38 & 336794.59 & 16.4 \\
\hline & $\mathrm{CV}(\%)$ & & 22.78 & 13.8 \\
\hline & Mean & & 2548.08 & 29.35 \\
\hline \multirow{7}{*}{ 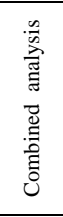 } & Gen & 19 & $2029446.79^{m}$ & $60.32^{* 3}$ \\
\hline & Env & 1 & $59554949.82^{* *}$ & $2136.42^{* *}$ \\
\hline & Rep (Env) & 4 & 369353.02 & 2.64 \\
\hline & Gen X Env & 19 & $369688.79^{*}$ & $28.35^{* *}$ \\
\hline & Residuals & 76 & 201709.5 & 9.88 \\
\hline & CVe $(\%)$ & & 24.36 & 12.5 \\
\hline & Mean & & 1843.6 & 25.13 \\
\hline & \multicolumn{4}{|c|}{ Dissimilarity between environments } \\
\hline \multicolumn{3}{|c|}{$\%$ of the simple part of $\mathrm{MS}_{\mathrm{GE}} \mathrm{E}_{\mathrm{j}}{ }^{\mathrm{G}}$} & \multicolumn{2}{|c|}{78.69} \\
\hline \multicolumn{3}{|c|}{$\%$ of the simple part of $\mathrm{MS} \mathrm{GE}_{\mathrm{jij}}{ }^{\prime} \mathrm{PE}$} & \multicolumn{2}{|c|}{22.41} \\
\hline
\end{tabular}

WS: water stress; WW: well-watered; Gen: genotype; Env: environment; Rep: replication; MS: mean square; CV: Coefficient of variation. GY: grain yield $\left(\mathrm{Kg} \cdot \mathrm{ha}^{-1}\right)$; PE: popping expansion $\left(\mathrm{g} . \mathrm{mL}^{-1}\right){ }^{*}$ and $* *$ are significant at $0.05 \%$ and $0.01 \%$ probability, by the $\mathrm{F}$ test, respectively.

\section{Agronomic potential of popcorn lines in WS and WW environments}

The GY means ranged from 400.00 to $1885.42 \mathrm{~kg} \cdot \mathrm{ha}^{-1}$ under WS and from 1318.75 to $3640.63 \mathrm{~kg} \cdot \mathrm{ha}^{-1}$ in the WW environment (Fig. 3). The group of lines with highest GY under WS contained the genotypes L54, L59, L76, P2, P3, P5, P6, P7, P8, and P9. In the WW environment, the same genotypes were present in the group of highest GY lines, along with L69 and P4; however, with exception of L54. The lines L61, L63, L65, and L75 constituted the group with lowest GY under WS.

The mean PE ranged from 16.33 to $28.33 \mathrm{~mL} . \mathrm{g}^{-1}$ and from 21.72 to $36.66 \mathrm{~mL} . \mathrm{g}^{-1}$ in the WS and WW environments, respectively (Fig. 3). Under WS, the mean PE was highest in lines L54, P1 and P5. Lines L61, L65, L69, P2, and P4 composed the group of lowest 
PEunder WS. The lines L54, L55, L59, L61, L70, L71, P1, P5, P7, P8, and P9 constituted the group with highest PE in the WW environment.

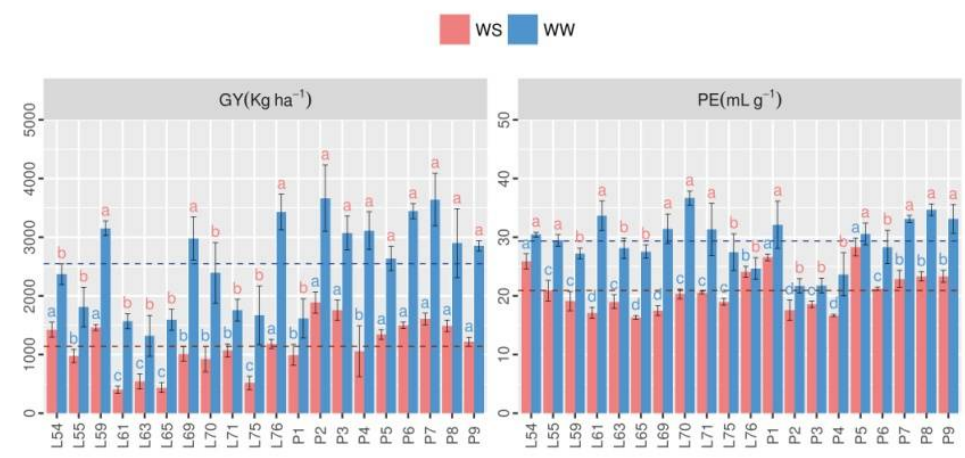

Figure 3. Agronomic potential of popcorn lines under WS and WW conditions.

\section{WS tolerance and agronomic water-use efficiency based on indices}

The Stress Tolerance Index (STI) (Fernandez, 1992) was defined as a useful tool to determine the maximum genotype yield under water stress and also the potential of water stress-tolerance. The mean STI was 0.49 for GY and 0.72 for PE (Fig 4). The best genotypes are those with values exceeding the overall mean of the index, i.e., lines L54, L59, L76, P2, P3, P4, P5, P6, P7, P8, and P9 for GY, and lines L54, L70, L71, P1, P5, P7, P8, and P9 for PE.L54, P7, P8, and P9 were outstanding for both indices (Fig. 4).

The Stress Susceptibility Index (SSI) (Fischer, 1978) measures the yield stability of a given genotype in relation to the mean stability of the evaluated genotypes. An appropriate SSI is lower than one unit $(<1)$, a condition fulfilled by the lines L54, L55, L59, L71, P1, P2, P3, P5, and P8 for GY; and L54, L76, P1, P2, P3, P5, and P6 for PE. The mean SSI was 1.00 for GY and 0.97 for PE (Fig. 4). The Stability Index (SIN) (Bouslama and Schapaugh, 1984) assesses the yield stability of genotypes under stress and non-stress conditions. A SIN value is adequate when closest to the unit (1), as it reveals higher values for the studied trait in the stress environment. Thus, the values were highest in the lines L54, L55, L59, L71, P1, P2, P3, P5, and P8 for GY; and L54, L76, P1, P2, P3, P5, and P6 for PE (Fig. 4).

The Drought Resistance Index (DRI) (Blum, 1988) is useful to identify genotypes that produce high yields under water stress and adequate water conditions, and also considers the percentage loss between environments. The mean DRI for GY was 0.47 and 0.52 for PE. This index is based on two estimates, i.e., higher values for the trait studied under stress and the loss percentage in the environment with adequate conditions. Adequate values are superior to the overall mean, condition fulfilled by lines L54, L59, L71, P1, P2, P3, P5, P6, P7, and P8 for GY; and by L54, L76, P1, P2, P3, P5, P6, P7, P8, and P9 for PE (Fig. 4).

Agronomic water-use efficiency (WUE) is used if the unit of water applied during the cultivation period is known. This index is related to the productivity of water applied in terms of agricultural yield, i.e., weight of harvested crop per liter of water used. Thus, WUE 
is the ratio between the amount of produced (or harvested) dry matter and the water volume applied to the crop (irrigation + precipitation, in this case). Thus, ideal genotypes are those with a high WUE index, e.g., lines L54, L59, L69, P2, P3, P4, P5, P6, P7, P8, and P9 for GY; and lines L54, L70, P1, P5, P7, P8, and P9 for PE (Fig. 4).

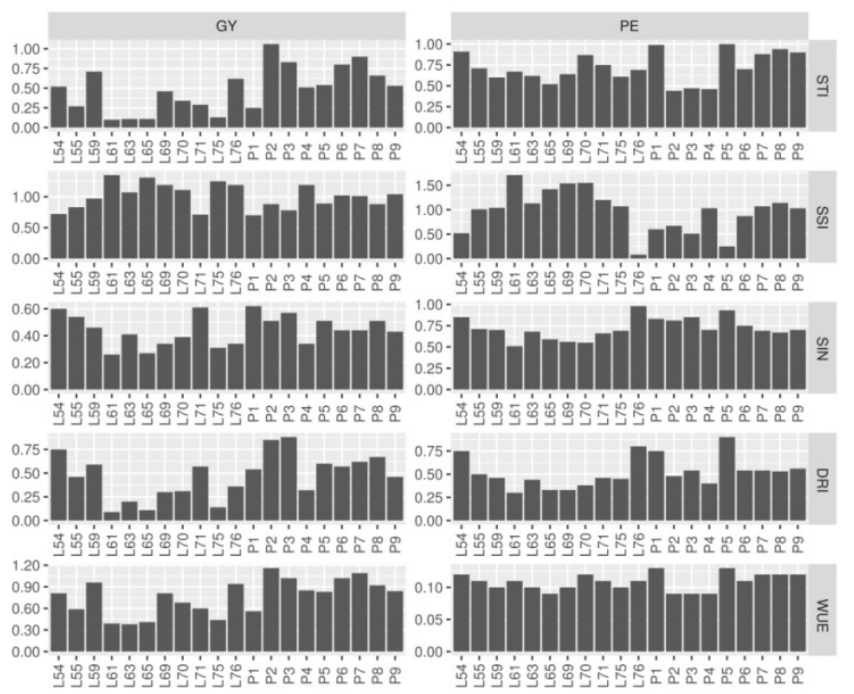

Figure 4. Indices of water-stress tolerance and agronomic water-use efficiency in popcorn lines.

\section{Potential lines for breeding}

\section{Grain yield}

Principal component analysis highlighted the dispersion of the indices, underlying the formation of four groups (Fig 5), based on the Euclidean distance. The values of the first principal component corresponded to $99.18 \%(\mathrm{PC} 1=72.17 \%+\mathrm{PC} 2=27.01 \%)$.

Group I (GI-GY) was formed by lines L59, P2, P3, P6, and P7 (Fig. 5). These lines have high GY means in WS and WW environments and, because they have high agronomic water use efficiency, they also have higher STI and WUE estimates. Group II (GII-GY) included lines L54, L55, L71, P1, P5, and P8 (Fig. 5). This second group is characterized by higher yield stability (SIN closer to one) and lower WS susceptibility (lower SSI), however, the GY means are not the highest. The GI-GY and GII-GY are similar in terms of DRI, given the intermediate position in the diagram of this index between the groups (Fig. 5).

Group III (GIII-GY) was composed of lines L69, L70, L76, P4, and P9 (Fig. 5). These genotypes are characterized by low stability (SIN far from unit) and high WS susceptibility (SSI>1), but have intermediate WUE and STI values, for having high GY means under WW conditions. Group IV (GIV-GY) was composed of lines L61, L63, L65, and L75 (Fig. 5). This group is similar to GIII-GY in view of the low GY under WS and high loss percentages in comparison with WW conditions. Consequently, the stress resistance (lower DRI), agronomic water-use efficiency (lower WUE) and finally the yield stability (SIN far from one) of the lines of GIV-GY were all low. 


\section{Popping expansion}

Principal component analysis (PCA) resulted in an index dispersion underlying the formation of five groups (Fig. 5), estimated by WARD's clustering method, based on the Euclidean distance. The values of the first principal components corresponded to $99.47 \%(\mathrm{PC} 1=66.24 \%+\mathrm{PC} 2=33.23 \%)$.

Group I (GI-PE) was composed of P7, P8 and P9 (Fig. 5). Under WW conditions, this group is characterized by lines with high PE, which is however considerably reduced under WS. Due to the high PE mean under WW conditions, the STI, DRI and WUE values were high, in spite of the low SSI and SIN values. Group II (GII-PE) contained lines L61, L69, and L70 (Fig. 5). These genotypes, similarly to the previous group, are characterized by high PE means in the WW environment, but with highest losses under WS. Thus, due to the low PE means under WS, this group is characterized mainly by stress susceptibility (SSI <1), low stability (SIN below one) and low DRI.

Group III (GIII-PE) was formed by the lines L55, L59, L63, L65, L71, L75, P4, and P6 (Fig 5). This set of genotypes is characterized by intermediate PE means under WW conditions, which are significantly reduced under WS. Consequently, the stress resistance of the lines GIII-PE (lower DRI) and stability (SIN below unit) was low in both environments. Group IV (GIV-PE) comprised lines P2 and P3 (Fig 5). The mean $\mathrm{PE}$ of this group was low in both environments, although the proportional loss between environments was reduced. In GIV-PE, the values of SSI and SIN were high and those of the other indices low.

Group V (GV-PE) consisted of lines L54, L76, P1, and P5 (Fig 5). This group is characterized by high $\mathrm{PE}$ means in both environments and in general, low proportional losses. Thus, in this group, DRI, SIN and WUE were high and SSI low.
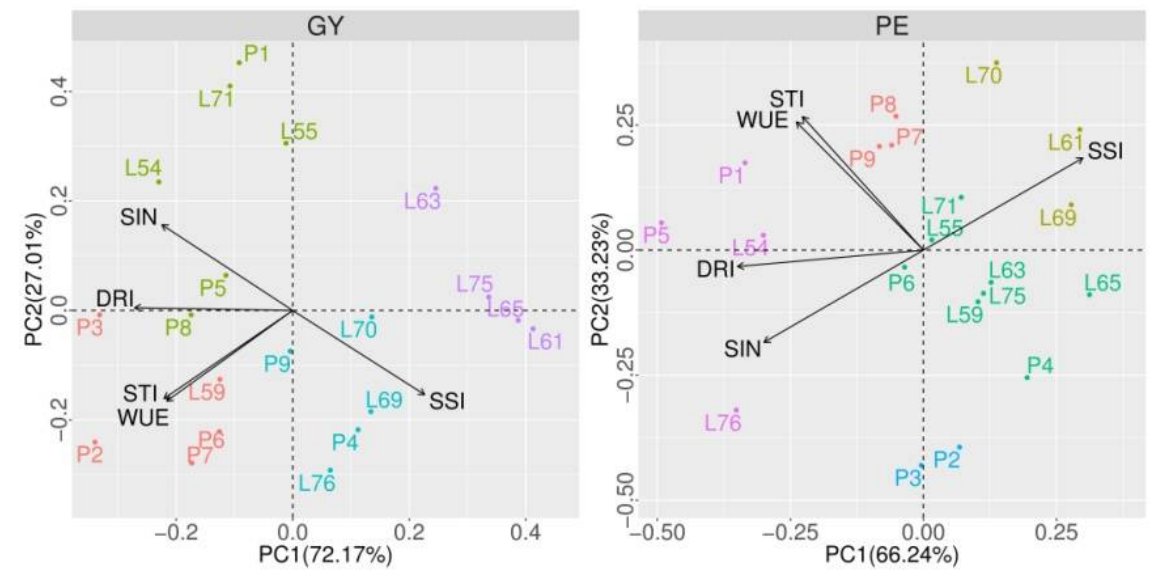

Figure 5. Grouping of popcorn lines, based on principal component analysis, by means of indices of water stress tolerance and agronomic water-use efficiency for grain yield (GY) and popping expansion (PE) in popcorn lines. 


\section{Genetic diversity measured by EST-SSR markers for potential crosses and inheritance studies}

By the EST-SSR markers two groups were formed, namely: GI-SSR, composed of L54, P1, P2, P4, P5, P6, P7, P8, and P9; and GII-SSR, formed by L55, L59, L61, L63, L65, L69, L70, L71, L75, L76, and P3 (Fig. 6). In general, the clusters coincided with the original variety of each line, except for L54 and P3. In this sense, the shortest genetic distances were detected between lines L70 x L71 (0.19), derived from variety BRS Angela and between lines P8 x P9 (0.23), derived from hybrid IAC112 (Fig. 6).

The lines that composed GI-GY (L59, P2, P3, P6, and P7) stood out for the indices STI and WUE and reached the highest GY means under WS and WW conditions, and with the greatest genetic distances between the following combinations: P3 x L59 (0.63), P6 x L59 (0.52) and P2 x L59 (0.51) (Fig 6). Phenotypically contrasting with GI-GY was Group IV (L61, L63, L65 and L75), characterized by low STI and WUE values. The longest genetic distances between the lines of these distinct groups were between P2 x L63 (0.67), P7 x L63 (0.61) and P3 x L61 (0.60) (Fig. 6).

GII-GY (L54, L55, L71, P1, P5 and P8) must be considered as the most WS tolerant lines and, based on the longest genetic distances, the following combinations were proposed: L55 x L71 ( 0.61), L71 x P5 (0.60) and L55 x P8 (0.58) (Fig 6). In phenotypical contrast with GII-GY, GIV-GY (L61, L63, L65, and L75) should be considered (Fig. 5). In this sense, the following combinations of L63 x P1 (0.72), L63 x P5 (0.70) and L63 x P8 (0.68) (Fig. 6) stand out with the longest genetic distances.

Based on the longest genetic distances between the lines with highest agronomic water-use efficiency (GI-GY) and WS tolerance (GII-GY), the following combinations are promising: L55 x P2 (0.63), L71 x P6 (0.62) and L71 x P3 (0.58) (Fig. 6).

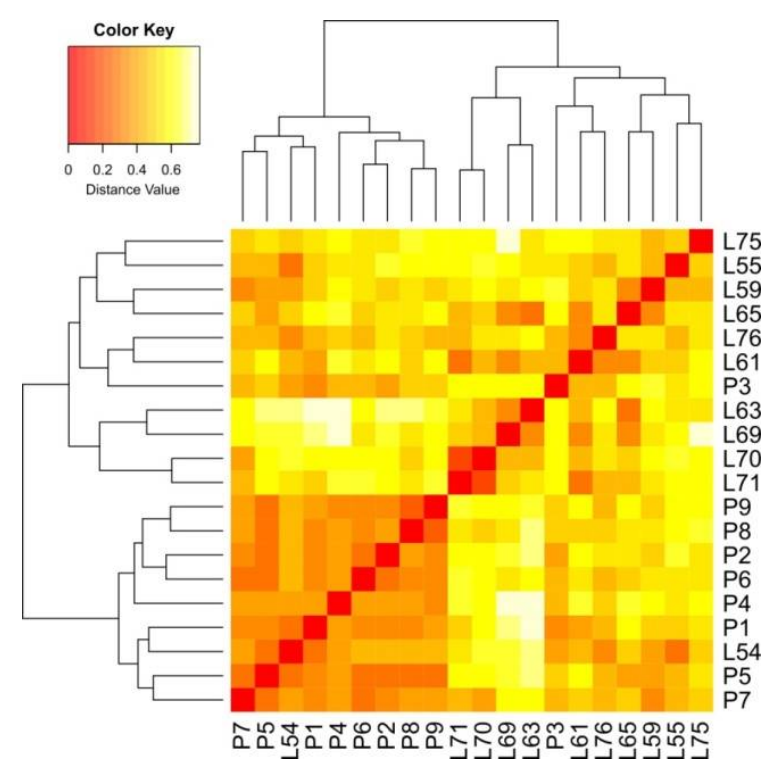

Figure 6. Dispersion diagram of genetic distances among 20 popcorn lines estimated by means of 15 EST-SSR markers. 


\section{DISCUSSION}

The existence of genetic variability in the popcorn lines was confirmed by the significant differences observed in the traits GY and PE, indicating possible genetic gains with selection. A differential response of popcorn lines to WS and WW environments is expected, due to the significant effect of the genotype-environment (GE) interaction. The GE interaction interferes with selection gains and hampers cultivar recommendation. In these cases, the formation of specific populations and hybrid combinations for particular environments is suggested (Hallauer et al., 2010). For field corn, several researchers have reported significant GE interactions for GY and its components (Dias et al., 2018).

In the dissimilarity analysis between the environments, trait GY had a simple type interaction, showing that the lines responded, to a lesser extent, to water stress, maintaining the ranking of positions. On the other hand, a complex interaction effect was stated for PE and, in this case, popcorn lines responded differently to the WS and WW environments, altering the rankings. For trait PE, the selection of superior genotypes should be environment-specific (Hallauer et al., 2010).

After 22 days of irrigation suspension (12 days after male flowering), soil water potential sensors installed at a depth of $0.2 \mathrm{~m}$ measured soil water tension values below the permanent wilting point $(-1.5 \mathrm{MPa})$. The difference of $78 \mathrm{~mm}$ water between the water regimes showed a significant reduction effect on GY and PE (Table 3). As an adaptive mechanism to WS, flowering is delayed for about 1.5 days (data not shown). The biological processes related to pollen viability, zygote formation and early grain filling are WSsensitive (Zinselmeier et al.,1995).

In the evaluated popcorn genotypes, a mean reduction of $55.29 \%$ in GY was recorded due to environmental stress during pre-anthesis until the beginning of grain filling. The water limitation must be long enough so that the plants can respond in a timely manner with acclimatization, which can be assessed in the final yield (Romano et al., 2011; Cairns JE et al., 2012). Bruce et al. (2002) mentioned a mean GY reduction of $\approx 50 \%$ as normal in genotypic evaluations under WS. For PE, the proportional decrease was $29.19 \%$. The expansion process is associated with the moisture content in the grain starch granules, which when heated $\left(\approx 180^{\circ} \mathrm{C}\right)$, exerts pressure on the pericarp, exposing the endosperm upon rupture (da Silva et al., 1993). This is the first report of PE reduction under WS and requires more detailed studies to understand this phenomenon.

Initially, to answer the question "Popcorn breeding for tolerance to water stress or for agronomic water-use efficiency?", the GY and PE means are observed concomitantly under WS and WW environments. Only line P5 had simultaneously higher means of GY and PE in both environments. However, when analyzing only the highest mean (WUE) of a given trait, the proportion of losses ( $\mathrm{T}$ ) between environments cannot be measured, requiring the use of indices. All indices used were highest in lines L54 and P5, and lowest for lines L61, L63, L65, and L75.

The line grouping and distribution of the indices for trait GY showed an association between STI and WUE, as well as an inverse relationship between SIN and SSI. The indices STI and WUE favor the establishment of a group with higher-yielding genotypes under both water regimes, the same that had a good WUE (GI-GY). On the other hand, the genotypes with the lowest percentage loss between environments (GII-GY), denominated tolerant, were not the same as those with a higher GY. Less productive genotypes tend to be tolerant 
to stress environments (Maia et al., 2011; Naghavi et al., 2013). The yield of tolerant genotypes is often moderate even under optimum growth conditions (Maia et al., 2011). According to Souza et al. (2008), selection should be performed for WUE, since the yields of genotypes are higher in both environments. Therefore, it can be assumed that exploiting genotypes with high WUE is the most acceptable option.

The indices used detected two aspects: higher mean values of a given trait (STI and WUE) and lower proportional loss rates (SSI and SIN). Among the indices used, DRI can be considered the most robust, since it simultaneously weighs mean values and proportional reduction. However, the acronym DRI appears to be incorrect, in particular in relation to the letter "R", indicating resistance. The term Drought Resilience Index is proposed, considered more appropriate. The DRI allowed the identification of maize genotypes that united high GY values under WS and WW conditions, considering the proportional reduction among the environments.

In some research publications, a conceptual paradox for the terms tolerance and efficiency is apparent. In these cases, genotypes with higher yields under WS are selected and are referred to as breeding for drought tolerance without estimating the reduction in relation to the ideal condition (Santos et al., 2003; Câmara et al., 2007; Teixeira et al., 2010). The selection based on the absolute yield under stress refers to the agronomic efficiency in the use of the resources. Clarifying the differences between the terms tolerance and agronomic efficiency in resource use implies in the selection of distinct genotypes for use in breeding. For Maia et al. (2011), due to the physiological mechanisms, simultaneous breeding for tolerance and efficient agronomic use of the resources seems to be contradictory.

In a scenario of global climate change, especially associated with the distribution of water resources, research is required to identify germplasm sources under limited water availability. Based on the results described here, it is possible to establish two strategies: identification of tolerance (T) sources (GII-GY) or efficiency in the use of this resource (GIGY). Our pool of popcorn lines, from different regions of Brazil, contains sufficient variability for both strategies.

Popcorn breeding for WS environments should address not only GY, but also essentially PE. The grouping of lines and distribution of the indices for PE, similar to that observed in GY, demonstrates the association between STI and WUE, as well as the inverse relationship between SIN and SSI. For PE, GV-PE was composed of genotypes with high DRI, with generally high mean PE in WS and WW environments and moderate proportional reduction, which was not observed for GY. However, there are possibilities for different breeding strategies, e.g., of breeding focused on Tor on WUE separately.

The dispersion of the groups for the traits GY and PE indicated an absence of correlation between WUE (more productive) and $\mathrm{T}$ (more stable), which allows the assumption that these traits are controlled by different genes. In a study on tolerance due to low nutrient stress in soil and nutritional efficiency in tropical maize, Maia et al. (2011) described a lack of relationship between tolerance and agronomic water-use efficiency and, therefore, claimed that these traits are governed by different genes. Thus, for WUE concomitantly with $\mathrm{T}$, there is a possibility of success with simultaneous selection based on selection indices weighing both tolerance and efficiency.

Given the absence of a relationship between WUE (more productive) and T (more stable), different responses to environmental improvement (supplementary irrigation) can be 
observed, namely: i) tolerant genotypes: capacity to maintain constant production, but which do not respond to environmental improvement; these genotypes may be alternatives to less technical or marginal agricultural environments, as well as for use in physiological studies; ii) genotypes with good agronomic water use efficiency: higher yields under both water regimes, however, more severe WS damage; these genotypes may be indicated for environments with a higher level of agricultural technology. For higher WUE genotypes, it is noted that small increases in resource availability result in a substantial yield increases (Maia et al., 2011), indicating that it is more promising to work with higher WUE genotypes. Other environmental factors that limit plant growth and development, e.g., temperature, high soil Al concentration, and salinity should be included in breeding programs for greater tolerance (Giaveno et al., 2007; Maia et al., 2011).

Strategic mechanisms are developed by plants to adapt to adverse environmental conditions (Trewavas, 2005), be it water-use efficiency or drought tolerance. From a physiological point of view, it is expected that for the most efficient genotypes, agronomical water use efficiency will not be the most tolerant to lack of the resource. Under adverse conditions, functional and structural traits of plant tissues, e.g., leaves and roots, are altered, which interferes with the maintenance of the plant, affecting yields (Sultan, 1995). However, the amount of metabolic resources available to stressed plants is limited (Zandalinas et al., 2018; Trewavas, 2005). Due to the reduced metabolic resources in stressed plants, the allocation and distribution of these compounds among the different plant structures is affected, with implications for each of the strategies adopted by the plant (Trewavas, 2005). In this way, according to Maia et al. (2011), with the increasing demand for resources to develop strategic mechanisms, plants can become inefficient in the use of resources in response to environmental challenges.

The groups with lowest loss percentage or highest agronomic efficiency water use are not the same for the traits GY and PE. In the literature published papers, a negative genetic correlation between these two traits is commonly observed, denoting, in the reciprocal sense, that direct selection for either one reduces the expression of the other (Hallauer et al., 2010). The explanation for the contrariness between the groups formed for GY and PE is due to the prevailing dominance gene effects for the first trait and, additive effects for the second (Pereira and Amaral, 2001; Lima et al., 2016; Schwantes et al., 2018). Given the distinct genetic control for the traits GY and PE, different breeding strategies are required.

Based on the diversity analysis with molecular markers, it is possible to propose that the hybrid combinations P3 x L59, P6 x L59 and P2 x L59 are indicated for allelic complementation for higher WUE (higher means) for GY. However, for trait PE, as it is controlled by additive genes, in which $\mathrm{F}_{1}$ presents the mean of the parents, the parents selected for crosses should have high PE values. Thus, line P7of group GI-GY becomes attractive, for expressing the highest mean PE. Therefore, the best hybrid combination for WUE, with higher PE volume, is a combination of the parents L59 x P7. Line L59 is characterized by intermediate PE means; and line P7, by high STI, DRI and WUE values for this trait.

Along this same line of reasoning, seeking allelic complementation for WS tolerance (GII) for GY, we recommend the combinations between the parents L55 x L71, L71 x P5 and L55 x P8. However, line P1 has high GY means in both environments. Therefore, the best hybrid combination for T, associated with higher PE volume, is the 
combination between the parents L55 x P1. Line L55 is characterized by intermediate PE means and line P1 by high DRI, SIN and WUE.

To explore genetic complementarity for tolerance (GII-GY) and higher agronomic water-use efficiency (GI-GY) concomitantly, the most promising cross is L71 x P6, because the mean PE was the highest among the possible hybrid combinations. We identified a group of lines from population BRS Angela (L61, L63, L65) that are phenotypically contrasting with the lines with highest $\mathrm{T}$ and WUE and also genetically divergent. Crosses between these lines should be used for inheritance studies.

\section{CONCLUSIONS}

There is genetic variability for both agronomic water-use efficiency and tolerance to low water availability, allowing different lines of research in breeding programs. The relationship between tolerance and agronomic water-use efficiency for the traits grain yield and popping expansion appears to be inversely related. Selection for water use efficiency seems to be the most adequate strategy to breed the most productive genotypes under both water regimes.

\section{ACKNOWLEDGMENTS}

Scholarships were provided by FAPERJ to MFR and by the Improvement of Higher Education Personnel program (CAPES) to CCGC. This study was financed in part by CAPES - Finance Code 001.

\section{REFERENCES}

Adebayo MA, Menkir A, Blay E, et al. (2014) Genetic analysis of drought tolerance in adapted $\times$ exotic crosses of maize inbred lines under managed stress conditions. Euphytica. 196:261-270.

Blum A. (1988). Plant breeding for stress environments. CRC Press. Florida, USA.

Bouslama M, Schapaugh W. (1984) Stress tolerance in soybean. Part 1: evaluation of three screening techniques for heat and drought tolerance. Crop Sci. 24: 933-937.

Bruce WB, Edmeades GO, Barker TC. (2002). Molecular and physiological approaches to maize improvement for drought tolerance. J. Exp. Bot. 53: 13-25.

Cairns JE, Sanchez C, Vargas M, et al (2012). Dissecting Maize Productivity: Ideotypes Associated with Grain Yield under Drought Stress and Well-watered Conditions. J.Integr. Plant Biol. 54:1007-1020.

Câmara TMM, Bento DAV, Alves GF, Santos MF, et al. (2007). Parâmetros genéticos de caracteres relacionados à tolerância à deficiência hídrica em milho tropical. Bragantia. 66: 595-603.

Cruz CD, Castoldi F. (1991). Decomposição da interação genótipo x ambientes em partes simples e complexa. Ceres. 38: 422-430.

Cruz CD, Regazzi AJ, Carneiro PCS. (2012). Modelos Biométricos Aplicados ao Melhoramento Genético. Viçosa: UFV. da Silva WJ, Vidal BC, Martins MEQ, Vargas H, et al. (1993). What makes popcorn pop. Nature. 362: 417-417.

Daher RF, Pereira MG, Pereira AV, Amaral Jr. AT. (2002). Genetic divergence among elephantgrass cultivars assessed by RAPD markers in composit samples. Sci. Agric. 59: 623-627.

Dias KODG, Gezan SA, Guimarães CT, Parentoni SN, et al. (2018). Estimating Genotype $\times$ Environment Interaction for and Genetic Correlations among Drought Tolerance Traits in Maize via Factor Analytic Multiplicative Mixed Models. Crop Sci. 58: 72.

Doyle, J.J., Doyle JL. (1990). Isolation of plant DNA from fresh tissue. Focus. 12: 13-15.

Fernandez G. (1992). Effective selection criteria for assessing plant stress tolerance. Proceedings of the International Symposium on Adaptation of Vegetables and other Food Crops in Temperature and Water Stress. Taiwan; pp. 257270.

Fischer R, Maurer R. (1978). Drought resistance in spring wheat cultivars. I. Grainyield responses. Aust. J. Agric. Res. 29: 897. 
Giaveno CD, Ribeiro RV, Souza GM, Oliveira RF. Screening of tropical maize for salt stress tolerance. (2007). Crop Breed Appl. Biotechnol. 7: 304-313.

Gower JC. (1971). A General Coefficient of Similarity and Some of Its Properties. Biometrics. 27: 857.

Hallauer AR, Miranda Filho JB, Carena MJ. (2010). Quantitative genetics in maize breeding. Springer, New York, 663p.

Jákli B, Hauer-Jákli M, Böttcher F, Meyer zurMüdehorst J, et al. (2018). Leaf, canopy and agronomic water-use efficiency of field-grown sugar beet in response to potassium fertilization. J. Agron. Crop Sci. 204: 99-110.

Kamara AY, Menkir A, Badu-Apraku B, Ibikunle O. (2003). The influence of drought stress on growth, yield and yield components of selected maize genotypes. J. Agric. Sci. 141: 43-50.

Lima VJ, do Amaral Junior AT, Kamphorst SH, et al (2016). Combining ability of S3 progenies for key agronomic traits in popcorn: comparison of testers in top-crosses. Genet. Mol. Res. 15:1-14.

Maechler M, Rousseeuw P, Struyf A, Hubert, M, et al. (2017). Cluster Analysis Basics and Extensions. R package version 2.0.6.

Mageto EK, Makumbi D, Njoroge K, Nyankanga R (2017). Genetic analysis of early-maturing maize ( Zea Mays L.) inbred lines under stress and nonstress conditions. J. Crop Improv. 31:560-588.

Maia C, DoVale JC, Fritsche-Neto R, Cavatte PC, et al. (2011). The difference between breeding for nutrient use efficiency and for nutrient stress tolerance. Crop Breed Appl. Biotechnol. 11: 270-275.

Mendes WS, Drews TA, Medeiros JC, Rosa JD, et al. (2017). Development and productivity of maize in response to spatial arrangement under semiarid condition of Northeastern Brazil. Aust. J. Crop Sci. 11: 313-321.

Miti, F; Tongoona, P; Derera J. (2010). $\mathrm{S}_{1}$ selection of local maize landraces for low soil nitrogen tolerance in Zambia. African J. Plant Sci. 4: 67-81.

Mitra J. (2001). Genetics and genetic improvement of drought resistance in crop plants. Curr. Sci. 80: 758-763.

Naghavi MR, Pour AA. Khalili M. (2013). Evaluation of Drought Tolerance Indices for Screening Some of Corn (Zea mays L.) Cultivars under Environmental Conditions. Not Sci. Biol. 5: 388-393.

Pena GF, Amaral Junior AT, Ribeiro RM, Ramos HC, et al. (2015). Inference of genetic diversity in popcorn S3 progenies. Genet. Mol. Res.: GMR, 15 .

Pereira, MG, Amaral Júnior, AT. (2001). Estimation of Genetic Components in Popcorn Based on the Nested Design. Crop Breed Appl. Biotechnol. 1: 3-10.

Peters GP, Marland G, Le Quéré C, Boden T, et al. (2011). Rapid growth in CO2 emissions after the 2008-2009 global financial crisis. Nat. Clim. Chang. 2: 2-4.

R Core Team. ( 2017). R: A language and environment for statistical computing [Internet]. Vienna, Austria: R Foundation for Statistical Computing.

Romano G, Zia S, Spreer W, Sanchez C, et al. (2011). Use of thermography for high throughput phenotyping of tropical maize adaptation in water stress. Comput. Electron. Agric. Elsevier B.V.; 79: 67-74.

Santos MX, Andrade CLT, Oliveira AC, Leite CEP, et al. (2003). Comportamento de Híbridos de Milho Selecionados e não Selecionados para Asi sob Estresse de Água no Florescimento e no Enchimento de Grãos. Rev. Bras. Milho e Sorgo. 2: 71-81.

SayadiMaazou A-R, Tu J, Qiu J, Liu Z. (2016). Breeding for Drought Tolerance in Maize. Am. J. Plant Sci. 7: 18581870.

Schwantes IA, do Amaral Júnior AT, Vivas M, et al. (2018). Inheritance of resistance to Fusarium ear rot in popcorn. Crop Breed Appl. Biotechnol. 18:81-88.

Sharopova N, McMullen MD, Schultz L, Schroeder S, et al. (2002). Development and mapping of SSR markers for maize. Plant Mol. Biol. 48: 463-481.

Souza LV, Miranda GV, Galvão JCC, Eckert FR, et al. (2008). Genetic control of grain yield and nitrogen use efficiency in tropical maize. Pesqui. Agropecuária Bras. 43: 1517-1523.

Sultan SE. (1995). Phenotypic plasticity and plant adaptation. Acta Bot. Neerl. 44: 363-383.

Suzuki N, Rivero RM, Shulaev V, Blumwald E, et al. (2014). Abiotic and biotic stress combinations. New Phytol. 203: 32-43.

Tambussi EA, Bort J, Araus JL. (2007). Water use efficiency in C3 cereals under Mediterranean conditions: a review of physiological aspects. Ann. Appl. Biol. 150: 307-321.

Teixeira FF, Gomide RL, Albuquerque PEP, Andrade CLT, et al. (2010). Evaluation of maize core collection for drought tolerance. Crop Breed Appl. Biotechnol. 10: 312-320.

Trewavas A. (2005). Green plants as intelligent organisms. Trends Plant Sci. 10: 413-419.

Warnes, GR, Bolker B, Bonebakker L, Gentleman R,et al. (2016). gplots: Various R Programming Tools for Plotting Data [Internet]. R package version 3.0.1.

Zandalinas SI, Mittler R, Balfagón D, et al. (2018). Plant adaptations to the combination of drought and high temperatures. Physiol. Plant. 162:2-12.

Zia S, Romano G, Spreer W, Sanchez C, et al. (2013). Infrared Thermal Imaging as a Rapid Tool for Identifying WaterStress Tolerant Maize Genotypes of Different Phenology. J. Agron. Crop Sci. 199: 75-84.

Zinselmeier SA, Lauer MJ, Boyer JS. (1995). Reversing Drought-Induced Losses in Grain Yield: Sucrose Maintains Embryo Growth in Maize. Crop Sci. 35: 1390. 